\title{
Stator- and Rotor-Flux-Based Deadbeat Direct Torque Control of Induction Machines
}

\author{
Barbara H. Kenny, Member, IEEE, and Robert D. Lorenz, Fellow, IEEE
}

\begin{abstract}
A new deadbeat type of direct torque control (DTC) is proposed, analyzed, and experimentally verified in this paper. The control is based on stator and rotor flux as state variables. This choice of state variables allows a graphical representation which is transparent and insightful. The graphical solution shows the effects of realistic considerations such as voltage and current limits. A position- and speed-sensorless implementation of the control, based on the self-sensing signal injection technique, is also demonstrated experimentally for low-speed operation. The paper first develops the new deadbeat DTC methodology and graphical representation of the new algorithm. It then evaluates feasibility via simulation and experimentally demonstrates performance of the new method with a laboratory prototype including the sensorless methods.
\end{abstract}

Index Terms-Deadbeat control, direct torque control (DTC), induction motor, position-sensorless control.

\section{INTRODUCTION}

D IRECT torque control (DTC) of induction machines has increasingly become an alternative to field-orientation methods [1], [2]. The classical method of DTC involves the use of a lookup table to select voltage vectors based on torque and stator flux magnitude error [3]. There is no current regulator, no pulsewidth modulation (PWM), nor reference frame transformations as in field orientation. With appropriately high sample rates this leads to fast torque response and low ripple. In addition, at operating conditions where the stator flux vector can be estimated accurately from the terminal voltage and current, this technique is also position and speed sensorless. However, at extremely low and zero speeds, the sensorless implementation of this technique suffers the same performance degradation as any control technique based on the estimate of stator flux using only fundamental voltage and current.

An alternative method of DTC is based on the deadbeat (inverse) solution to the machine equations [4]-[7]. The deadbeat solution is similar to the classical DTC method in that it controls torque and stator flux directly, without an intermediate current loop. It is different, however, in the calculation of the voltage vector to be applied to the machine. In the deadbeat solution, an inverse model is used to calculate the theoretical voltage vector

Paper IPCSD 03-022, presented at the 2001 Industry Applications Society Annual Meeting, Chicago, IL, September 30-October 5, and approved for publication in the IEEE TRANSACTIONS ON INDUSTRY APPLICATIONS by the Industrial Drives Committee of the IEEE Industry Applications Society. Manuscript submitted for review July 1, 2002 and released for publication April 14, 2003.

B. H. Kenny is with the NASA Glenn Research Center, Cleveland, OH 44135 USA (e-mail: Barbara.Kenny@ grc.nasa.gov).

R. D. Lorenz is with the Departments of Mechanical Engineering and Electrical and Computer Engineering, University of Wisconsin, Madison, WI 53706 USA (e-mail: lorenz@engr.wisc.edu).

Digital Object Identifier 10.1109/TIA.2003.813727 needed to move the machine torque and stator flux to the desired values in one sample period. This voltage vector is then synthesized over the sample period by the use of PWM modulation techniques. However, the calculation of the voltage vector requires the solution of a quadratic equation with several parameter dependent coefficients. Insight into the operation of the control is lost with a purely algebraic approach to the solution of the quadratic equations.

This paper presents a new DTC strategy where stator and rotor flux are chosen as state variables in the deadbeat solution [8], [9]. The use of stator and rotor flux as state variables, represented in the stator-flux-oriented synchronous reference frame, allows the construction of an intuitive graphical depiction of the necessary voltage vector to achieve the commanded torque and stator flux magnitude values in one time step. The graphical depiction changes as operating conditions or parameters vary; thus, the resulting change in the necessary voltage vector can clearly be seen. Conversely, the impact of the selection of the wrong voltage vector on both the stator flux and torque errors can also be seen. In addition, practical operating limits can be shown on the same graph, thus presenting a good visualization of the current and voltage limitations. It is further shown that the structure of this control strategy is suitable for use with the self-sensing position estimation technique [10]. This enables low- and zero-speed position- and speed-sensorless DTC.

\section{Formulation of A STATOR-Rotor-Flux DeAdBEAT CONTROL ALGORITHM}

The state equations for the induction machine, using the stator and rotor flux as state variables in the stationary reference frame, are as follows [11]:

$$
\begin{aligned}
p \boldsymbol{\lambda}_{q d s} & =\mathbf{V}_{q d s}-\left(\frac{R_{s}}{\sigma L_{s}}\right) \boldsymbol{\lambda}_{q d s}+\left(\frac{R_{s} L_{m}}{\sigma L_{s} L_{r}}\right) \boldsymbol{\lambda}_{q d r} \\
p \boldsymbol{\lambda}_{q d r} & =\left(-\frac{R_{r}}{\sigma L_{r}}+j \omega_{r}\right) \boldsymbol{\lambda}_{q d r}+\left(\frac{L_{m} R_{r}}{L_{r} \sigma L_{s}}\right) \boldsymbol{\lambda}_{q d s} \\
\tau_{e} & =\frac{3 P L_{m}}{4 \sigma L_{s} L_{r}}\left\{\boldsymbol{\lambda}_{q d s} \times \boldsymbol{\lambda}_{q d r}\right\} .
\end{aligned}
$$

A discrete-time form of (1)-(3) is shown in (4)-(6) that is valid for small values of the sample time $t_{s}$ for which the rotor speed $\omega_{r}$ changes negligibly [8], [9].

$$
\begin{aligned}
& \lambda_{q d s}(k+1)-\lambda_{q d s}(k) \\
& \quad=\mathbf{V}_{q d s}(k) t_{s}-\left(\frac{R_{s}}{\sigma L_{s}}\right) \boldsymbol{\lambda}_{q d s}(k) t_{s}+\left(\frac{R_{s} L_{m}}{\sigma L_{s} L_{r}}\right) \boldsymbol{\lambda}_{q d r}(k) t_{s}
\end{aligned}
$$




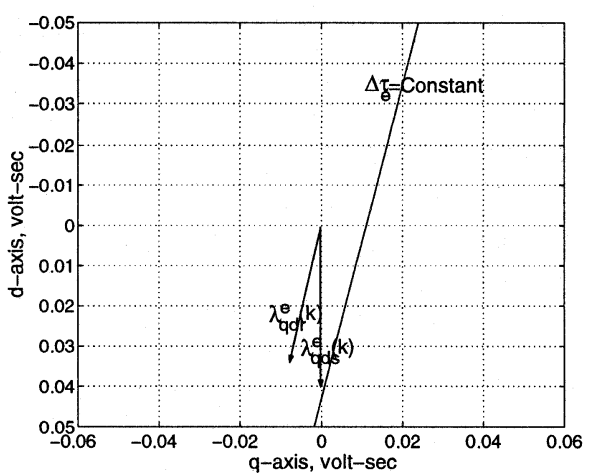

Fig. 1. Constant $\Delta \tau_{e}(k)$ line.

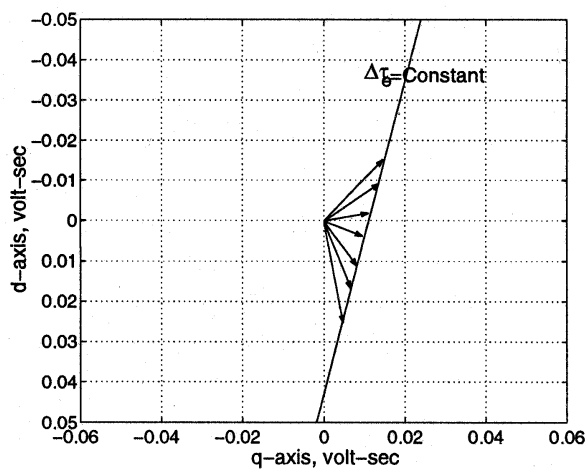

Fig. 2. Graphical representation of multiple voltage vectors for machine torque change $\Delta \tau_{e}$.

$$
\begin{aligned}
& \boldsymbol{\lambda}_{q d r}(k+1)-\boldsymbol{\lambda}_{q d r}(k) \\
& \quad=\left(-\frac{R_{r}}{\sigma L_{r}}+j \omega_{r}\right) \boldsymbol{\lambda}_{q d r}(k) t_{s}+\left(\frac{L_{m} R_{r}}{L_{r} \sigma L_{s}}\right) \boldsymbol{\lambda}_{q d s}(k) t_{s} \\
& \tau_{e}(k+1)=\frac{3 P L_{m}}{4 \sigma L_{s} L_{r}}\left\{\boldsymbol{\lambda}_{q d s}(k+1) \times \boldsymbol{\lambda}_{q d r}(k+1)\right\} .
\end{aligned}
$$

Equations (4)-(6) can be combined to form an expression for the change in torque, $\Delta \tau_{e}(k)=\tau_{e}(k+1)-\tau_{e}(k)$. If the $d$ axis of the excitation reference frame is aligned with the stator flux and the terms proportional to $t_{s}^{2}$ are neglected, a very useful relationship results as shown in (7) [8], [9]

$$
\begin{aligned}
\frac{\Delta \tau_{e}(k)}{t_{s}}= & \tau_{e}(k)\left(\frac{-R_{r}}{\sigma L_{r}}-\frac{R_{s}}{\sigma L_{s}}\right)+\frac{3 P L_{m}}{4 \sigma L_{s} L_{r}}\left\{-\omega_{r} \lambda_{d s}^{e}(k)\right. \\
& \left.\times \lambda_{d r}^{e}(k)+V_{q s}^{e}(k) \lambda_{d r}^{e}(k)-V_{d s}^{e}(k) \lambda_{q r}^{e}(k)\right\} .
\end{aligned}
$$

Equation (7) can be rearranged as follows to show the linear relationship between $V_{q s}^{e}(k) t_{s}$ and $V_{d s}^{e}(k) t_{s}$ values which can be used to provide a given value of $\Delta \tau_{e}(k)$ :

$$
\begin{array}{r}
V_{q s}^{e}(k) t_{s}=\frac{4 \sigma L_{s} L_{r}}{3 P L_{m} \lambda_{d r}^{e}(k)}\left(\Delta \tau_{e}(k)+\tau_{e}(k)\left(\frac{R_{r}}{\sigma L_{r}}+\frac{R_{s}}{\sigma L_{s}}\right) t_{s}\right) \\
+\omega_{r} \lambda_{d s}^{e}(k) t_{s}+V_{d s}^{e}(k) t_{s} \frac{\lambda_{q r}^{e}(k)}{\lambda_{d r}^{e}(k)} .
\end{array}
$$

If (8) is plotted in a $d-q$ plane with $V_{d s}^{e}(k) t_{s}$ and $V_{q s}^{e}(k) t_{s}$ as the $d$ - and $q$-axes variables, respectively, the voltage loci for a given $\Delta \tau_{e}(k)$ is a straight line. This line is parallel to the rotor

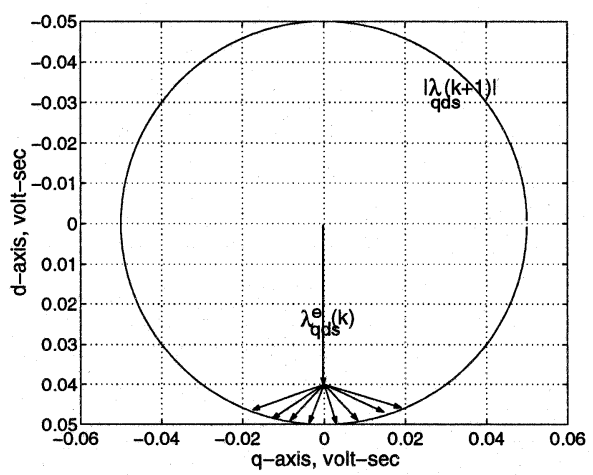

Fig. 3. Graphical representation of multiple voltage vectors for flux magnitude increase.

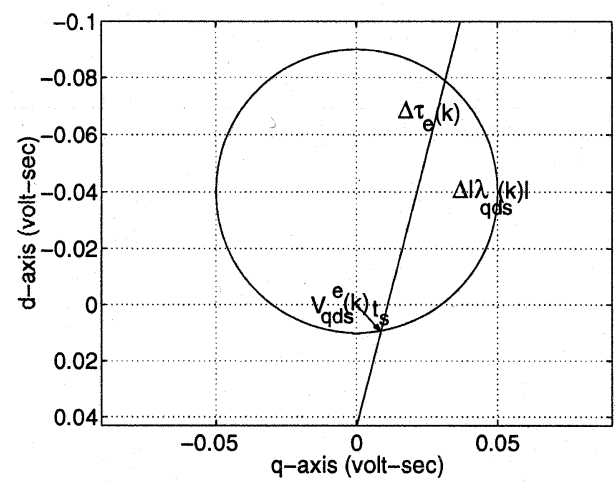

Fig. 4. Graphical representation of a voltage vector which satisfies both $\Delta \tau_{e}(k)$ and $\Delta\left|\boldsymbol{\lambda}_{q d s}(k)\right|$ requirements.

flux vector $\boldsymbol{\lambda}_{q d r}^{e}(k)$, as shown in Fig. 1 [8], [9]. (Figs. 1-6 use the convention $f_{q d}=f_{q}-j f_{d}$.)

One representation of the multiple possible voltage (volt-second) vectors $\left[\begin{array}{lll}V_{q s}^{e}(k) t_{s} & V_{d s}^{e}(k) t_{s}\end{array}\right]$ that could yield the desired change in motor torque $\Delta \tau_{e}(k)$ is shown in Fig. 2.

From (4), the discrete-time expression for stator flux, neglecting stator resistance, is

$$
\boldsymbol{\lambda}_{q d s}(k+1)-\boldsymbol{\lambda}_{q d s}(k)=\mathbf{V}_{q d s}(k) t_{s}
$$

Equation (9) can also be shown graphically. Fig. 3 shows the plot of $\left|\boldsymbol{\lambda}_{q d s}(k+1)\right|$ and $\lambda_{d s}^{e}(k)$, where $\lambda_{d s}^{e}(k)=\left|\boldsymbol{\lambda}_{q d s}(k)\right|$. There are multiple voltage vectors, scaled by $t_{s}$, which will move the flux magnitude from a value of $\lambda_{d s}^{e}(k)$ to $\left|\boldsymbol{\lambda}_{q d s}(k+1)\right|$.

The flux circle shown in Fig. 3 can be redrawn to show the change in flux magnitude, $\Delta\left|\lambda_{q d s}(k)\right|=\left|\boldsymbol{\lambda}_{q d s}(k+1)\right|-\lambda_{d s}^{e}(k)$, where $\lambda_{d s}^{e}(k)=\left|\lambda_{q d s}(k)\right|$. This circle is centered on the present value of stator flux $\lambda_{d s}^{e}(k)$ with a radius equal to the magnitude $\left|\lambda_{q d s}(k+1)\right|$. This adjustment means that the possible voltage (volt-second) vector loci shown in Figs. 2 and 3 now all begin at the origin of the plot. Thus, for a given set of $\Delta \tau_{e}(k)$ and $\Delta\left|\lambda_{q d s}(k)\right|$ requirements, the voltage vector which will solve both conditions simultaneously can be determined from the intersection of the $\Delta \tau_{e}(k)$ line and the $\Delta\left|\boldsymbol{\lambda}_{q d s}(k)\right|$ circle. This is shown in Fig. 4.

The equation defining the $\Delta\left|\boldsymbol{\lambda}_{q d s}(k)\right|$ circle is given in (10). The voltage vector which will solve both the $\Delta \tau_{e}(k)$ and the $\Delta\left|\lambda_{q d s}(k)\right|$ requirements can be found mathematically from the 


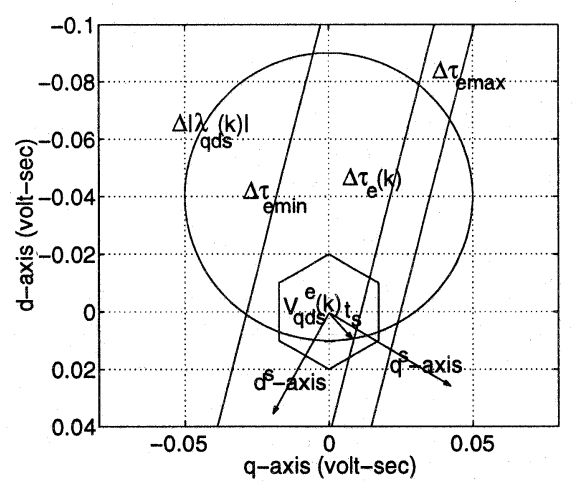

Fig. 5. Graphical representation of voltage and current limits on the possible voltage vector solutions.

simultaneous solution of (8) and (10), or graphically as shown in Fig. 4

$$
\left(V_{q s}^{e}(k) t_{s}\right)^{2}+\left(V_{d s}^{e}(k) t_{s}-\lambda_{d s}^{e}(k)\right)^{2}=\left|\lambda_{q d s}(k+1)\right|^{2} .
$$

The solution $\mathbf{V}_{q d s}^{e}(k) t_{s}$ to the intersection of the $\Delta \tau_{e}(k)$ line and the $\Delta\left|\boldsymbol{\lambda}_{q d s}(k)\right|$ circle is bounded by the available dc-bus voltage, the sample time, and the inverter current limit. The range of voltages that can be synthesized from a two-level inverter can be represented as a hexagon in the $d-q$ plane [11]. Thus, the bound for $\mathbf{V}_{q d s}^{e}(k) t_{s}$ is a hexagon with sides equal to $2 / 3 V_{d c} t_{s}$ as shown in Fig. 5. The hexagon is shown at a static position in Fig. 5 but it actually rotates at the synchronous speed because the figure is in the synchronous reference frame.

Fig. 5 also shows the maximum and minimum values of $\Delta \tau_{e}$ based on a steady-state solution using the inverter current limit $\left|\mathbf{i}_{q d s m a x}\right|$ and the present value of $\tau_{e}(k)$. The maximum torque can be calculated as a function of the stator flux magnitude and the maximum stator current as follows. For a stator flux oriented system, the torque can be expressed as

$$
\tau_{e}=\frac{3 P}{4} \lambda_{d s} i_{q s}
$$

In terms of current magnitude, this becomes

$$
\tau_{e}= \pm \frac{3 P}{4} \lambda_{d s} \sqrt{\left|\mathbf{i}_{q d s}\right|^{2}-i_{d s}^{2}} .
$$

The variables $i_{d s}$ and $\lambda_{d s}$ can be related through the slip frequency by using the following two relationships which are given in [11]:

$$
\begin{aligned}
\boldsymbol{\lambda}_{q d s} & =L_{\sigma s} \mathbf{i}_{q d s}+\frac{L_{m}}{L_{r}} \boldsymbol{\lambda}_{q d r} \\
p \boldsymbol{\lambda}_{q d r} & =\left(\frac{-R_{r}}{L_{r}}-j \omega_{e}+j \omega_{r}\right)\left(\boldsymbol{\lambda}_{q d r}\right)+\left(\frac{L_{m} R_{r}}{L_{r}}\right) \mathbf{i}_{q d s} .
\end{aligned}
$$

Assuming stator flux orientation and steady-state conditions, (13) and (14) can be used to form an expression for $i_{d s}$ in terms of the stator current magnitude and the stator flux

$$
i_{d s}=\frac{L_{\sigma s} L_{s}\left|\mathbf{i}_{q d s}\right|^{2}+\lambda_{d s}^{2}}{\lambda_{d s} L_{s}(1+\sigma)} .
$$

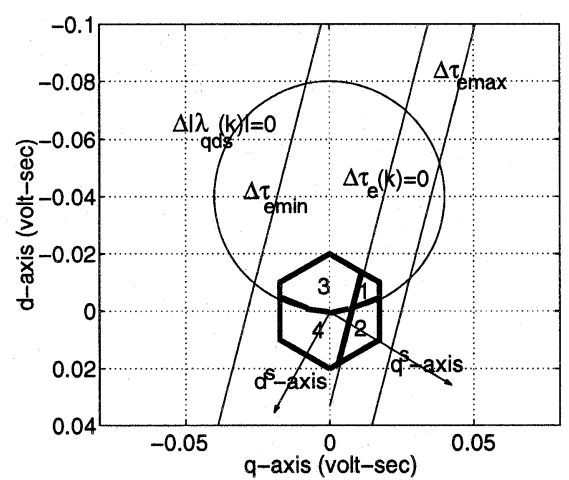

Fig. 6. Graphical representation of operating regions for steady-state conditions.

Equation (15) can be substituted into (12) to find a condition on the maximum torque that is possible for a specified maximum stator current magnitude and stator flux value

$\tau_{e \max }= \pm \frac{3 P}{4} \lambda_{d s} \sqrt{\mathbf{i}_{q d s \max }^{2}-\left(\frac{L_{\sigma s} L_{s}\left|\mathbf{i}_{q d s \max }\right|^{2}+\lambda_{d s}^{2}}{\lambda_{d s} L_{s}(1+\sigma)}\right)^{2}}$

Finally, using (16) and the present value of torque, the maximum and minimum change in torque can be calculated

$$
\begin{aligned}
\Delta \tau_{e \max } & =+\tau_{e \max }-\tau_{e}(k) \\
\Delta \tau_{e \min } & =-\tau_{e \max }-\tau_{e}(k) .
\end{aligned}
$$

It is interesting to consider the plot for a steady-state condition $\left(\Delta \tau_{e}(k)=0, \Delta\left|\boldsymbol{\lambda}_{q d s}(k)\right|=0\right)$. This is shown in Fig. 6 .

Any $\Delta \tau_{e}$ lines to the right of the $\Delta \tau_{e}(k)=0$ line represent an increase in torque. Any $\Delta \tau_{e}$ lines to the left of $\Delta \tau_{e}(k)=0$ represent a decrease in torque. Any $\Delta\left|\boldsymbol{\lambda}_{q d s}(k)\right|$ circles within the $\Delta\left|\boldsymbol{\lambda}_{q d s}(k)\right|=0$ circle represent a decrease in stator flux magnitude. Any $\Delta\left|\boldsymbol{\lambda}_{q d s}(k)\right|$ circles outside $\Delta\left|\boldsymbol{\lambda}_{q d s}(k)\right|=0$ represent an increase in flux. Thus, it is seen that there are four operating regions within the hexagon as shown:

1) increase torque, decrease flux;

2) increase torque, increase flux;

3) decrease torque, decrease flux;

4) decrease torque, increase flux.

The standard table lookup method of DTC allows only the voltage vectors represented by the vertices of the hexagon (plus the two zero vectors) to be selected for the duration of the sample period. However, if a PWM technique is used, an average voltage vector over the sample period can be synthesized which lies anywhere within the hexagon.

A control algorithm can be developed based on (8), (10), and Fig. 4 if $\Delta \tau_{e}(k)$ is set equal to $\tau_{e}(k)^{*}-\tau_{e}(k)$ and $\Delta\left|\boldsymbol{\lambda}_{q d s}(k)\right|=$ $\left|\lambda_{q d s}(k)\right|^{*}-\lambda_{d s}^{e}(k)$. The voltage vector calculated by the control algorithm is the intersection of the $\Delta \tau_{e}(k)^{*}$ line with the $\Delta\left|\boldsymbol{\lambda}_{q d s}(k)\right|^{*}$ flux magnitude circle which is the solution of (8) and (10). The voltage vector can be synthesized using spacevector modulation (SVM) techniques to calculate the inverter switch duty cycles [12]. Fig. 7 shows the block diagram of the 


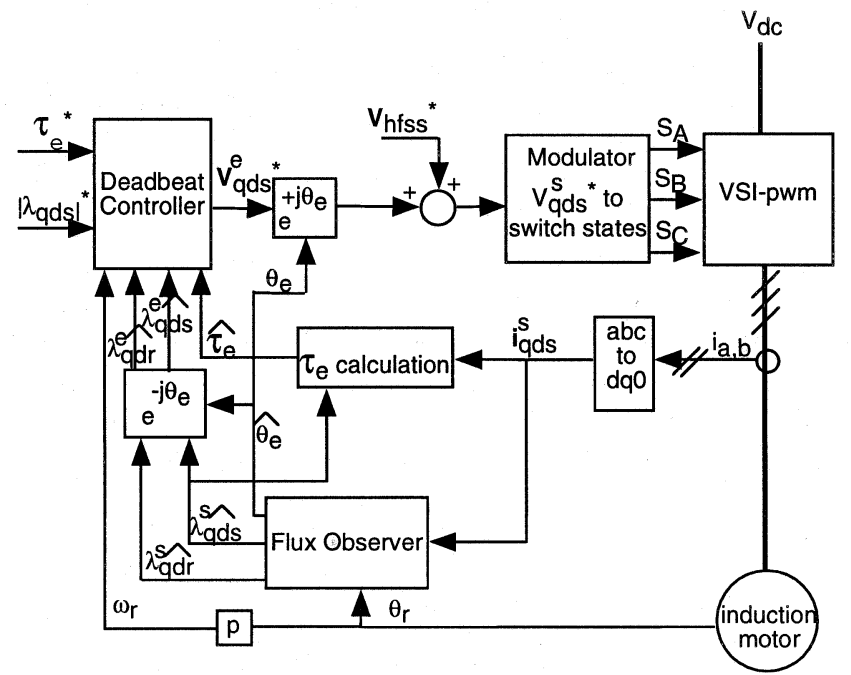

Fig. 7. Proposed deadbeat control block diagram.

control system in which the proposed algorithm was implemented. (The additional voltage command $V_{h f s s}^{*}(k)$ is only necessary for the self-sensing position and speed estimation as explained in Section V.)

The control algorithm first tests if $\Delta \tau_{e}(k)^{*}$ is within the limits of $\Delta \tau_{e \max }$ and $\Delta \tau_{e \min }$. If it is not, $\Delta \tau_{e}(k)^{*}$ is set equal to the closest limit. There are then three possibilities for the voltage vector solution: the $\Delta \tau_{e}(k)^{*}$ line and the $\Delta\left|\lambda_{q d s}(k)\right|^{*}$ circle intersect within the allowable voltage hexagon, the $\Delta \tau_{e}(k)^{*}$ line and the $\Delta\left|\boldsymbol{\lambda}_{q d s}(k)\right|^{*}$ circle intersect outside the allowable hexagon, or the $\Delta \tau_{e}(k)^{*}$ line and the $\Delta\left|\lambda_{q d s}(k)\right|^{*}$ circle do not intersect.

If the $\Delta \tau_{e}(k)^{*}$ line and the $\Delta\left|\lambda_{q d s}(k)\right|^{*}$ circle intersect within the allowable voltage hexagon, the solution is the voltage vector defined by the intersection point closest to the origin and is implemented using space vector modulation.

If the $\Delta \tau_{e}(k)^{*}$ line and the $\Delta\left|\boldsymbol{\lambda}_{q d s}(k)\right|^{*}$ circle intersect outside of the allowable voltage hexagon, the desired voltage vector is again calculated; however, the magnitude is larger than what is available given the dc-bus voltage. The magnitude of the voltage vector is reduced by the SVM algorithm until it lies on the hexagon boundary. This reduced-magnitude voltage vector is then applied.

If the $\Delta \tau_{e}(k)^{*}$ line and the $\Delta\left|\boldsymbol{\lambda}_{q d s}(k)\right|^{*}$ circle do not intersect, this is an indication that a large change in torque is required. In this case, the maximum voltage vector in the direction of maximum change in torque is applied. This is the voltage vector perpendicular to the $\Delta \tau_{e}(k)^{*}$ line with the maximum magnitude allowed by the hexagon.

\section{Simulation Results}

The experimental motor used in this paper is a specially designed high-speed (rated speed just over $23000 \mathrm{r} / \mathrm{min}$ ) induction machine for a NASA electromechanical actuator research project. The parameter values are given in the Appendix. The simulation conditions are set to match the experimental conditions. Experimentally, the machine was limited to $10000 \mathrm{r} / \mathrm{min}$

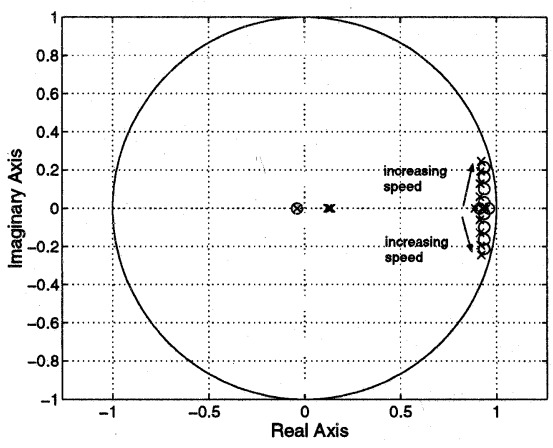

Fig. 8. Pole-zero plot for $100-\mu$ s sample time and no calculation delay.

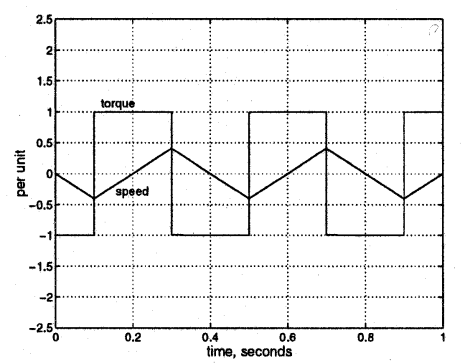

Fig. 9. Torque and speed response for a $100-\mu$ s sample time and no calculation delay.

or less due to concern about the condition of the bearings. All of the simulations neglect the PWM switching harmonics.

The controller was analyzed in two ways. First, to investigate its small-signal stability, the operating point model was formed using the Matlab LTI function. To form the operating point model, the mechanical dynamics were neglected (constant speed) and perfect flux estimation was assumed (the flux observer was not included in the operating point model). Pole-zero migration plots were then created for a range of speeds from low speed to rated speed (180-23000 r/min) at the rated torque and rated flux operating point.

The second analysis was based on a time-domain nonlinear model simulation of the complete system including the flux observer and the mechanical dynamics. The speed, torque, and stator flux responses to a square-wave torque command are shown.

Fig. 8 shows the pole-zero migration plot from the LTI analysis for a $100-\mu$ s sample time (used experimentally). The result is seen to approximate the expected deadbeat response but there is not exact pole-zero cancellation and the free pole is not exactly at the origin. This improves as a smaller and smaller sample time is used. (The NASA motor has relatively small time constants as can be seen in the Appendix.)

The time-domain results shown in Figs. 9 and 10 demonstrate good performance. The torque response is seen to be a square wave as expected. Fig. 10 also shows the effect of neglecting the resistance in deriving (9). For a stator resistance equal to zero, the stator flux is at the commanded value of 1 per unit. However, the stator flux magnitude is slightly reduced from the commanded value for a stator resistance of $0.18 \Omega$.

In the ideal case, it is assumed that the command values for the manipulated variables $\left(V_{q s}^{s^{*}}(k), V_{d s}^{s^{*}}(k)\right)$ are calculated in 


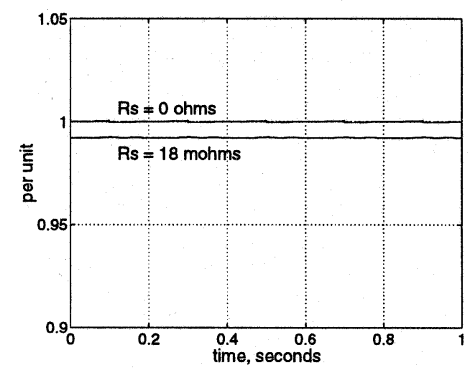

Fig. 10. Stator flux magnitude for $100-\mu$ s sample time, no calculation delay, and two values of stator resistance.

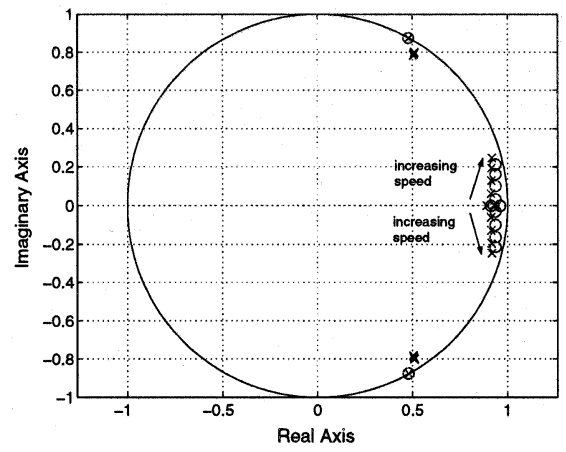

Fig. 11. Pole-zero plot for $100-\mu$ s sample time and one-step time delay.

zero time based on the present sampled value of the measured feedback variables $\left(i_{q s}(k), i_{d s}(k)\right)$. Realistically, however, there will be a calculation delay between when the feedback variables are measured and when the commanded values for the manipulated variables are updated because the required microprocessor calculations can not be done in zero time.

The command values for the manipulated variables $V_{q s}^{s^{*}}(k)$ and $V_{d s}^{s^{*}}(k)$ ultimately become pulsewidth commands for the gate drives in the inverter. In this implementation, the sampling time is synchronized with the PWM generation. Thus, the PWM timers are updated at the same time as the feedback variables are measured. This results in a one-step time delay for the control because the PWM timers are updated based on the calculations from the feedback data of the previous sample.

The effect of this one-step time delay can be seen in the pole-zero plot of Fig. 11. The free pole and the pole-zero cancellation pair that were at or near the origin in Fig. 8 have now moved to the edge of the unit circle. The time-domain simulations also show a more oscillatory response as seen in Figs. 12 and 13.

For a similar deadbeat type of controller, [5] shows that a modification to $\Delta \tau_{e}(k)^{*}$ and $\Delta\left|\boldsymbol{\lambda}_{q d s}(k)\right|^{*}$ will move the system poles to lie further within the unit circle and reduce the oscillations. A similar derivation can be shown here. To include the one-step time delay, (7) can be expressed as shown in (19)

$$
\begin{aligned}
& \frac{\tau_{e}(k+2)-\tau_{e}(k+1)}{t_{s}} \\
& =\tau_{e}(k+1)\left(\frac{-R_{r}}{\sigma L_{r}}-\frac{R_{s}}{\sigma L_{s}}\right)+\frac{3 P L_{m}}{4 \sigma L_{s} L_{r}} \\
& \quad \times\left\{-\omega_{r} \lambda_{d s}^{e}(k+1) \lambda_{d r}^{e}(k+1)+V_{q s}^{e}(k)^{*} \lambda_{d r}^{e}(k+1)\right. \\
& \left.\quad-V_{d s}^{e}(k)^{*} \lambda_{q r}^{e}(k+1)\right\} .
\end{aligned}
$$

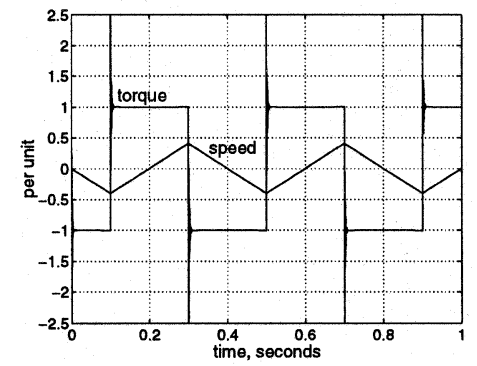

Fig. 12. Torque and speed response for a $100-\mu$ sec sample time and a one time step calculation delay.

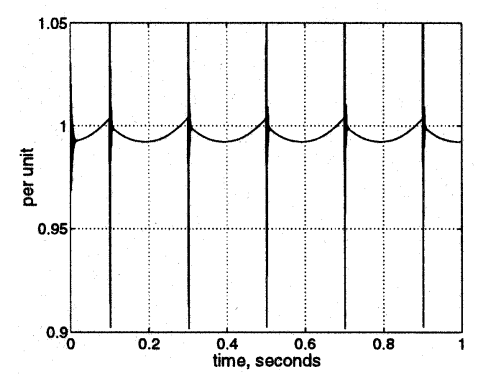

Fig. 13. Stator flux magnitude for $100-\mu$ s sample time and a one time step calculation delay.

From (8), the expression for the control variables $V_{q s}^{e^{*}}(k)$ and $V_{d s}^{e^{*}}(k)$ is

$$
\begin{aligned}
& V_{q s}^{e^{*}}(k) t_{s}-V_{d s}^{e^{*}}(k) t_{s} \frac{\lambda_{q r}^{e}(k)}{\lambda_{d r}^{e}(k)} \\
& =\omega_{r} \lambda_{d s}^{e}(k) t_{s}+\frac{4 \sigma L_{s} L_{r}}{3 P L_{m} \lambda_{d r}^{e}(k)} \\
& \quad \times\left(\tau_{e}(k)^{*}-\tau_{e}(k)+\tau_{e}(k)\left(\frac{R_{r}}{\sigma L_{r}}+\frac{R_{s}}{\sigma L_{s}}\right) t_{s}\right) .
\end{aligned}
$$

Under constant stator flux operation and assuming that $\lambda_{q d r}^{e}(k+1) \approx \boldsymbol{\lambda}_{q d r}^{e}(k),(19)$ and (20) can be combined to form an approximate relationship between the commanded torque and the actual torque as shown in (21)

$$
\begin{aligned}
\tau_{e}(k+2) & -\tau_{e}(k+1)=\tau_{e}(k+1)\left(\frac{-R_{r}}{\sigma L_{r}}-\frac{R_{s}}{\sigma L_{s}}\right) t_{s} \\
& +\tau_{e}(k)^{*}-\tau_{e}(k)+\tau_{e}(k)\left(\frac{R_{r}}{\sigma L_{r}}+\frac{R_{s}}{\sigma L_{s}}\right) t_{s} .
\end{aligned}
$$

From (21), the characteristic equation of this simplified transfer function is

$$
\begin{array}{r}
z^{2}-z\left(1-\left(\frac{R_{r}}{\sigma L_{r}}+\frac{R_{s}}{\sigma L_{s}}\right) t_{s}\right)+\left(1-\left(\frac{R_{r}}{\sigma L_{r}}+\frac{R_{s}}{\sigma L_{s}}\right) t_{s}\right) \\
=0 .
\end{array}
$$

The magnitude of the poles of (22) is given in (23). For small values of $t_{s}$, these poles can be seen to lie close to the unit circle boundary. This indicates that the deadbeat controller is very sensitive to the one-step time delay.

$$
\left|p_{1}\right|=\left|p_{2}\right|=\sqrt{\left(1-\left(\frac{R_{r}}{\sigma L_{r}}+\frac{R_{s}}{\sigma L_{s}}\right) t_{s}\right)} .
$$


One way to reduce the sensitivity of the controller to the one-step calculation delay is to relax the response required. Instead of a deadbeat response, an exponential response with a small time constant can be defined. The controller with the exponential response is much less sensitive to the one-step calculation delay [13].

If the commanded change in torque $\Delta \tau_{e}(k)^{*}$ in (8) and the commanded change in flux magnitude $\Delta\left|\lambda_{q d s}(k)\right|^{*}$ in (10) are reduced by a factor $C$, as shown in (24) and (25), the result is an exponential response. This can be seen by substituting the commanded values given in (26) into (7). The result is shown in (27)

$$
\begin{aligned}
\Delta \tau_{e}(k)^{*}= & C\left(\tau_{e}(k)^{*}-\tau_{e}(k)\right) \\
\Delta\left|\boldsymbol{\lambda}_{q d s}(k)\right|^{*}= & C\left(\left|\boldsymbol{\lambda}_{q d s}(k)\right|^{*}-\left|\boldsymbol{\lambda}_{q d s}(k)\right|\right) \\
V_{q s}^{e^{*}}(k) t_{s} & -V_{d s}^{e^{*}}(k) t_{s} \frac{\lambda_{q r}^{e}(k)}{\lambda_{d r}^{e}(k)} \\
= & \omega_{r} \lambda_{d s}^{e}(k) t_{s}+\frac{4 \sigma L_{s} L_{r}}{3 P L_{m} \lambda_{d r}^{e}(k)} \\
& \times\left(\tau_{e}(k)^{*}-\tau_{e}(k)+\tau_{e}(k)\left(\frac{R_{r}}{\sigma L_{r}}+\frac{R_{s}}{\sigma L_{s}}\right) t_{s}\right)
\end{aligned}
$$

$$
\tau_{e}(k+1)=C \tau_{e}(k)^{*}+\tau_{e}(k)(1-C) .
$$

Taking the $z$ transform of (27) results in the expected transfer function for an exponential response [14]

$$
\frac{\tau_{e}(z)}{\tau_{e}(z)^{*}}=\frac{C}{z-(1-C)} .
$$

To see the effect of the one-step calculation delay, (26) is substituted into (18) instead of (7). The result is given in (29) with the same assumptions given previously-that is, $\boldsymbol{\lambda}_{q d r}^{e}(k+$ $1) \approx \lambda_{q d s}^{e}(k)$ and $\lambda_{q d r}^{e}(k+1) \approx \lambda_{q d r}^{e}(k)$

$$
\begin{gathered}
\tau_{e}(k+2)-\tau_{e}(k+1)=\tau_{e}(k+1)\left(\frac{-R_{r}}{\sigma L_{r}}-\frac{R_{s}}{\sigma L_{s}}\right) t_{s} \\
+C \tau_{e}(k)^{*}-C \tau_{e}(k)+\tau_{e}(k)\left(\frac{R_{r}}{\sigma L_{r}}+\frac{R_{s}}{\sigma L_{s}}\right) t_{s} .
\end{gathered}
$$

The $z$ transform of (29) results in the transfer function shown in (30)

$$
\begin{aligned}
& \frac{\tau_{e}(z)}{\tau_{e}(z)^{*}}= \\
& \frac{C}{z^{2}-z\left(1-\left(\frac{R_{r}}{\sigma L_{r}}+\frac{R_{s}}{\sigma L_{s}}\right) t_{s}\right)+\left(C-\left(\frac{R_{r}}{\sigma L_{r}}+\frac{R_{s}}{\sigma L_{s}}\right) t_{s}\right)} .
\end{aligned}
$$

The magnitude of the poles of the transfer function in (30) is shown in (31). It can be seen that for $C<1$ the poles will move

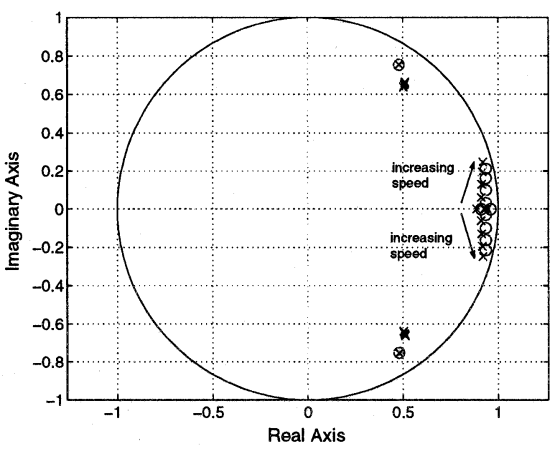

Fig. 14. Pole-zero plot for $100-\mu$ s sample time, one-step time delay, and $C=$ 0.8 .

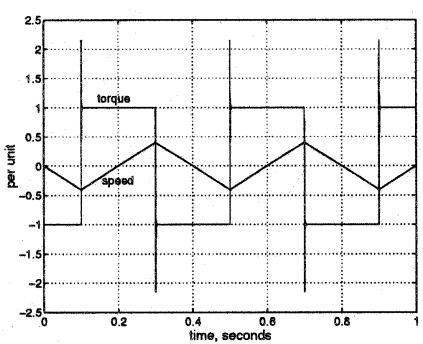

Fig. 15. Torque and speed response for a $100-\mu$ s sample time, one time step delay, and $C=0.8$.

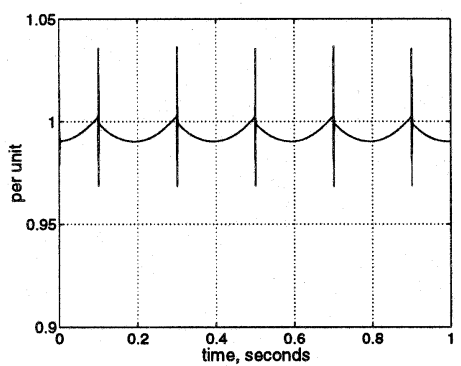

Fig. 16. Stator flux magnitude for a $100-\mu$ s sample time, one time step delay, and $C=0.8$.

further inside the unit circle and the response is expected to be less oscillatory

$$
\left|p_{1}\right|=\left|p_{2}\right|=\sqrt{\left(C-\left(\frac{R_{r}}{\sigma L_{r}}+\frac{R_{s}}{\sigma L_{s}}\right) t_{s}\right)} .
$$

The simulation results show this to be the case. Fig. 14 shows the LTI pole-zero migration plot for the controller with the commanded torque and stator flux magnitude values given by (24) and (25) respectively and $C=0.8$. The poles have clearly moved further inside the unit circle. The time-domain simulation results given in Figs. 15 and 16 show reduced overshoot and oscillation in the response.

\section{EXPERIMENTAL RESULTS}

The experimental test setup consisted of a dc power supply, an intelligent power module, a dSpace 1103 digital controller, and the NASA test motor. The results were captured using dSpace software. The data files were then plotted using Matlab. Two 


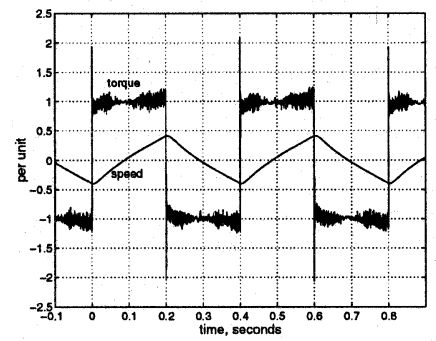

Fig. 17. Experimental results: torque and speed response for a $100-\mu$ s sample time, one time step delay, and $C=0.8$.

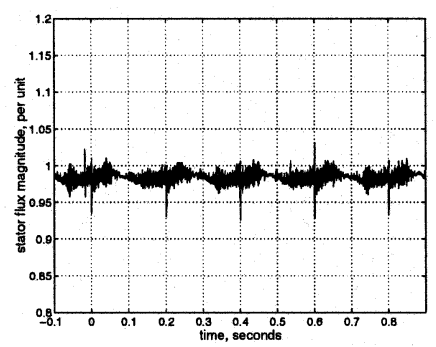

Fig. 18. Experimental results: stator flux magnitude for a $100-\mu$ s sample time, one time step delay, and $C=0.8$.

phase currents were measured and an encoder was used for position feedback. The position feedback was necessary because a flux observer based on the current model was used. (In Section V, the position feedback is provided by the self-sensing algorithm and the encoder information was used for comparison purposes only.) The speed was calculated in the controller using the position information. The torque and flux were estimated in the controller using current and position information.

Figs. 17 and 18 show the torque, stator flux, and speed for the same conditions as in Figs. 15 and 16 in simulation.

In general, the response is as predicted. In the actual implementation, there is always a deadtime, or blanking time, in the inverter so that the two switches across a leg do not conduct at the same time. In addition, there is a voltage drop across the conducting devices. Both of these effects result in a lower voltage being applied to the machine than is actually commanded. This "deadtime voltage" can be calculated [15]

$$
V_{\text {deadtime }}=\frac{4 V_{d}}{3} e^{j(k-1)(\pi / 3)} .
$$

$V_{d}$ is the magnitude of the voltage due to the combination of the deadtime losses and the conduction losses and $k$ is the sector of the $d-q$ plane in which the current vector is located, $k=1,2 \ldots 6$. This controller was found to be sensitive to deadtime compensation as can be seen by comparing Figs. 19 and 20 (without deadtime) to Figs. 17 and 18 (with deadtime). It is seen that without deadtime compensation, both the torque and stator flux magnitudes were reduced.

\section{LOW-SPEED SENSORLESS IMPLEMENTATION}

To date, the self-sensing method has been demonstrated only with the field orientation method of control. The use of selfsensing in a deadbeat DTC offers the potential of full speed

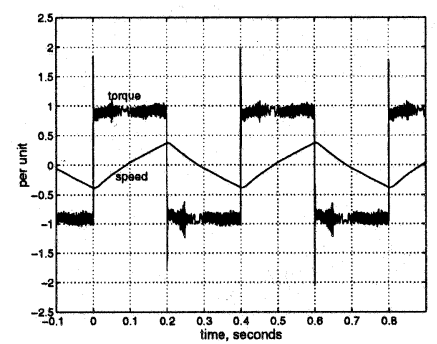

Fig. 19. Experimental results: torque and speed response for a $100-\mu$ s sample time, one time step delay, and $C=0.8$; no deadtime compensation.

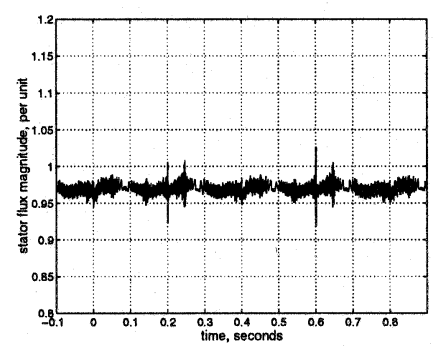

Fig. 20. Experimental results: stator flux magnitude for a $100-\mu$ s sample time, one time step delay, and $C=0.8$; no deadtime compensation.

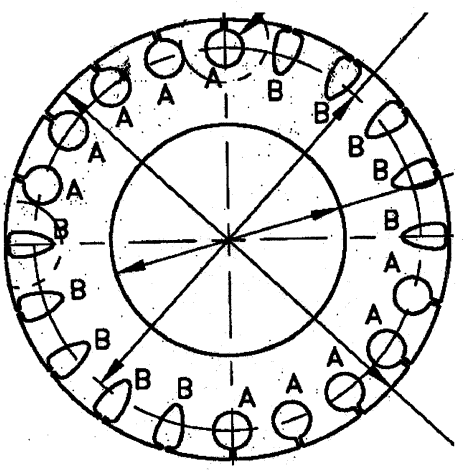

Fig. 21. Rotor cross section of NASA machine.

range sensorless operation with the flux estimate based on the back-electromotive force (EMF) method at higher speeds and on the self-sensing position estimate at lower speeds.

To estimate the rotor position angle $\theta_{r}$, the self-sensing technique requires a machine with a magnetic saliency related to the rotor position [10]. The NASA motor rotor was designed to produce a position-dependent magnetic saliency by changing the shape of the rotor bars as a function of position as shown in Fig. 21. (The rotor diameter is $1.3 \mathrm{in.}$.)

Additionally, the self-sensing technique requires a high-frequency carrier signal. The structure of a deadbeat type of DTC lends itself to the use of the self-sensing method of position estimation because the necessary high-frequency signal can be easily added to the fundamental voltage command as shown in Fig. 7. Thus, the commanded voltage into the PWM modulator will consist of the fundamental voltage command $\mathbf{V}_{q d s}^{s^{*}}(k)$ and the high-frequency voltage $V_{h f s s}^{*}(k)$. The resulting current is filtered as described in [14] and a signal at twice the rotor position is generated due to the position-dependent magnetic saliency. This signal can be tracked in a closed-loop saliency 


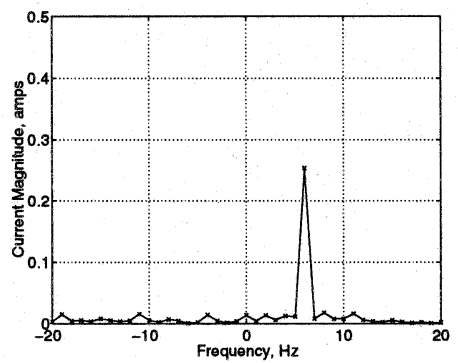

Fig. 22. Negative-sequence current spectra for constant-speed 3-Hz operation.

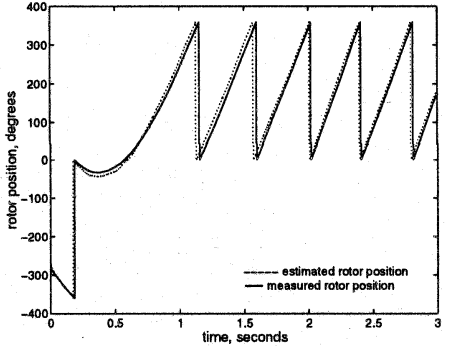

Fig. 23. Sensorless speed reversal of proposed controller with estimated rotor position and speed feedback based on self-sensing method.

image-tracking observer to produce position, velocity, acceleration, and disturbance torque estimates.

Figs. 22 and 23 show the results of a no-load closed-loop low-speed sensorless control using the self-sensing position estimate as feedback for the controller. Fig. 22 shows the spectra of the negative-sequence current (as defined in [16]) for a constant $3-\mathrm{Hz}$ speed. The component due to the rotor saliency is clearly visible at $6 \mathrm{~Hz}$. Fig. 23 shows a no-load speed reversal from $-3 \mathrm{~Hz}$ to $+3 \mathrm{~Hz}$.

\section{CONCLUSIONS}

This paper has developed a new deadbeat DTC method based on stator and rotor flux as state variables. This choice of state variables allows a clear graphical visualization of the voltage vector solution and the inverter operating limits.

The implementation of the proposed controller was evaluated experimentally and found to produce good results. The implementation issues which could limit performance were also evaluated.

The controller was found to be sensitive to the one-step time delay in the experimental implementation. The deadtime voltage drop, without appropriate compensation, was also found to reduce the torque and flux in the machine.

The structure of the proposed controller allows the addition of a high-frequency voltage vector to the commanded fundamental voltage vector. This allows the self-sensing method of position and speed estimation to be used thus demonstrating low, including zero speed, sensorless control.

\section{APPENDIX}

NASA motor: two-pole induction, $96 V_{L N}, 400 \mathrm{~Hz}, L_{m}=$ $1.9 \mathrm{e}-3 \mathrm{H}, L_{l s}=1.25 \mathrm{e}-4 \mathrm{H}, L_{l r}=1.25 \mathrm{e}-4 \mathrm{H}, R_{r}=0.105 \Omega$,
$R_{s}=0.09 \Omega, J=1.02 \mathrm{e}-4 \mathrm{~kg} \cdot \mathrm{m}^{2}, \tau_{e m}=1 \mathrm{~N} \cdot \mathrm{m}, \omega_{r}=23030$ $\mathrm{r} / \mathrm{min}$.

\section{REFERENCES}

[1] I. Takahashi and T. Noguchi, "A new quick-response and high-efficiency control strategy of an induction motor," IEEE Trans. Ind. Applicat., vol. IA-22, pp. 820-827, Sept./Oct. 1986.

[2] M. Depenbrock, "Direct self-control (DSC) of inverter-fed induction machine," IEEE Trans. Power Electron., vol. 3, pp. 420-429, Oct. 1988.

[3] P. Tiitinen, "The next generation motor control method, DTC, direct torque control," in Proc. 1996 Int. Conf. Power Electronics, Drives and Systems for Industrial Growth, New Delhi, India, Jan. 8-11, 1996, pp. $37-43$.

[4] T. Habetler, F. Profumo, M. Pastorelli, and L. Tolbert, "Direct torque control of induction machines using space vector modulation," IEEE Trans. Ind. Applicat., vol. 28, pp. 1045-1053, Sept./Oct. 1992.

[5] J. Maes and J. Melkebeek, "Discrete time direct torque control of induction motors using back-EMF measurement," in Conf. Rec. IEEE-IAS Annu. Meeting, St. Louis, MO, Oct. 12-15, 1998, pp. 407-414.

[6] F. Neves, B. Menezes, and S. Silva, "A deadbeat torque and flux field oriented controller for induction motor drives," in Proc. XII Brazilian Automatic Control Conf., vol. VI, Sept. 1998, pp. 2243-2247.

[7] G. Buja, M. Candela, and R. Menis, "A novel direct control scheme for SVM inverter-fed induction motor drives," in Proc. 1999 ISIE, Bled, Slovenia, pp. 1267-1272.

[8] D. Casadei, G. Serra, and A. Tani, "Analytical investigation of torque and flux ripple in DTC schemes for induction motors," in Proc. IEEE IECON'97, vol. 2, New Orleans, LA, Nov. 9-14, 1997, pp. 552-556.

[9] —- "Implementation of a direct torque control algorithm for induction motors based on a discrete space vector modulation," in Proc. IEEE PESC'98, vol. 2, Fukuoka, Japan, May 18-21, 1998, pp. 997-1003.

[10] P. L. Jansen and R. D. Lorenz, "Transducerless position and velocity estimation in induction and salient AC machines," IEEE Trans. Ind. Applicat., vol. 31, pp. 240-247, Mar./Apr. 1995.

[11] D. W. Novotny and T. A. Lipo, Vector Control and Dynamics of AC Drives. New York: Oxford Univ. Press, 1996.

[12] H. W. van der Broeck, H. C. Skudelny, and G. Stanke, "Analysis and realization of a pulse width modulator based on voltage space vectors," IEEE Trans. Ind. Applicat., vol. 24, pp. 142-150, Jan./Feb. 1988.

[13] B. H. Kenny, "Deadbeat direct torque control of induction machines using self-sensing at low and zero speeds," Ph.D. dissertation, Univ. Wisconsin, Madison, WI, 2001.

[14] J. G. Bollinger and N. A. Duffie, Computer Control of Machines and Processes. New York: Addison-Wesley, 1988.

[15] P. Vas, Sensorless Vector and Direct Torque Control. Oxford, U.K.: Oxford Univ. Press, 1998.

[16] M. Degner and R. Lorenz, "Using multiple saliencies for the estimation of flux, position, and velocity in AC machines," IEEE Trans. Ind. Applicat., vol. 34, pp. 1097-1104, Sept./Oct. 1998.

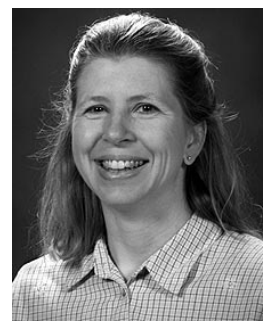

Barbara H. Kenny (M'82) was born in Syracuse, NY, in 1960. She received the B.S.E.E. degree from Cornell University, Ithaca, NY, in 1982, the M.S.E.E. degree from the University of Texas, Austin, in 1988, and the Ph.D. degree from the University of Wisconsin, Madison, in 2001.

From 1982 to 1986, she was on active duty with the U.S. Air Force in facilities engineering. Since 1989, she has been with the NASA Glenn Research Center, Cleveland, $\mathrm{OH}$, where she is currently a Research Engineer in the Electrical Systems Development Branch. Her main area of interest is in sensorless motor control for permanent-magnet and induction machines. Other areas of interest include high-speed machine design and control, and pulsewidth modulation techniques.

Dr. Kenny is a member of the IEEE Industry Applications, IEEE Power Electronics, IEEE Aerospace and Electronic Systems, and IEEE Women in Engineering Societies. She is a Registered Professional Engineer in the State of Virginia. 


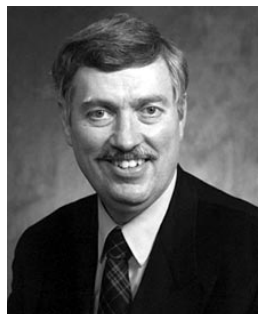

Robert D. Lorenz (S'83-M'84-SM'91-F'98)

received the B.S., M.S., and Ph.D. degrees from the University of Wisconsin, Madison, and the M.B.A. degree from the University of Rochester, Rochester, NY

Since 1984, he has been a member of the faculty of the University of Wisconsin, Madison, where he is the Mead Witter Foundation Consolidated Papers Professor of Controls Engineering in both the Department of Mechanical Engineering and the Department of Electrical and Computer Engineering. He is Co-Director of the Wisconsin Electric Machines and Power Electronics Consortium, which celebrated its 20th anniversary in 2001. It is the largest industrial research consortium on motor drives in the world. He is also the thrust leader for control and sensor integration in the Center for Power Electronic Systems, an NSF Engineering Research Center (ERC) which is a joint ERC with Virgina Polytechnic Institute and State University, Rensselaer Polytechnic Institute, University of Puerto Rico-Mayaguez, and North Carolina A\&T. From 1972 to 1982, he was a member of the research staff at the Gleason Works, Rochester NY, working principally on high-performance drives and synchronized motion control. He was a Visiting Research Professor in the Electrical Drives Group, Catholic University of Leuven, Leuven, Belgium, in the summer of 1989 and in the Power Electronics and Electrical Drives Institute, Technical University of Aachen, Aachen, Germany, in the summers of 1987, 1991, 1995, 1997, and 1999, where he also was the SEW Eurodrive Guest Professor from September 1, 2000 until July 7, 2001. In 1969-1970, he conducted Master thesis research in adaptive control of machine tools at the Technical University of Aachen. His current research interests include sensorless electromagnetic motor/actuator technologies, real-time signal processing and estimation techniques, precision multiaxis motion control, and ac/dc drive and high-precision machine control technologies. He has authored more than 160 published technical papers and is the holder of 16 patents, with two more pending.

Dr. Lorenz was the IEEE Industry Applications Society (IAS) President for 2001, a Distinguished Lecturer of the IAS for 2000/2001, immediate past Chair of the IAS Awards Department, and past Chairman of the IAS Industrial Drives Committee, and is a member of the IAS Industrial Drives, Electric Machines, Industrial Power Converter, and Industrial Automation and Control Committees. $\mathrm{He}$ is also the current Chair of the Periodicals Committee for the IEEE Technical Activities Board. He is a member of the IEEE Sensor Council AdCom. He is a Registered Professional Engineer in the States of New York and Wisconsin. He is also a member of the American Society of Mechanical Engineers, Instrument Society of America, and Society of Photo-Optical Instrumentation Engineers. $\mathrm{He}$ has won 15 prize paper awards. 